\title{
Combined photoacoustic and oblique-incidence diffuse reflectance system for quantitative photoacoustic imaging in turbid media
}

\author{
Janaka C. Ranasinghesagara \\ Roger J. Zemp \\ University of Alberta \\ Department of Electrical and Computer Engineering \\ 9107-116 Street \\ Edmonton, Alberta, Canada, T6G 2V4
}

\begin{abstract}
While photoacoustic imaging is capable of producing highresolution biomedical images with optical absorption contrast, optical property quantification has thus far remained challenging. One reason for this is that laser-induced photoacoustic signal amplitudes are proportional to not only the local optical absorption coefficient, but also the local laser fluence in the tissue. Unfortunately, local laser fluence is often unknown, but could possibly be estimated if local bulk tissue optical properties were known. One method to estimate tissue optical properties is a technique known as oblique incidence diffuse reflectance (OIR). We report on an integrated OIR and photoacoustic imaging system and demonstrate, using phantom experiments, improved ability to quantitatively estimate optical properties of a turbid medium. (- 2010 Society of Photo-Optical Instrumentation Engineers. [DOI: $10.1117 / 1.3470336]$
\end{abstract}

Keywords: photoacoustic; laser fluence; absorption coefficient; quantitative photoacoustics.

Paper 10100PR received Feb. 26, 2010; revised manuscript received May 5, 2010; accepted for publication Jun. 4, 2010; published online Aug. 2, 2010.

\section{Introduction}

Biomedical photoacoustic imaging is an emerging hybrid imaging technology producing images with high optical contrast and excellent spatial resolution. Because the contrast is due to optical absorption, a wide range of promising functional and molecular imaging methods are emerging. Examples include functional imaging of blood oxygen saturation, ${ }^{1-3}$ in vivo molecular imaging of gene expression, and molecular imaging using targeted contrast agents and nanoparticles. Noninvasive oxygen saturation measurements are valuable in various medical applications, such as monitoring brain oxygen saturation during coronary bypass surgery, ${ }^{4}$ understanding the effects of radiotherapy and chemotherapy on tumors, ${ }^{5}$ and monitoring the healing of wounds. ${ }^{6}$ Similarly, photoacoustic molecular imaging ${ }^{7-9}$ offers significant promise for visualizing disease progression noninvasively from a biochemical perspective.

In photoacoustic imaging, the pressure rise produced due to thermoelastic expansion is proportional to the Grüneisen parameter, the optical absorption coefficient, and the local laser fluence. At a constant temperature, the Grüneisen parameter for a given tissue type can be approximated as a constant. ${ }^{10}$ If we are able to compensate the effect of spatial and spectral variation in the laser fluence, normalized photoacoustic data can be used for quantitative photoacoustic imaging.

Address all correspondence to Roger J. Zemp, University of Alberta, Departmen of Electrical and Computer Engineering, 9107-116 Street, Edmonton, Alberta Canada, T6G 2V4. Tel: 780-492-1825; Fax: 780-492-1811; E-mail: zemp@ece.ualberta.ca
Several groups have tried to compensate local laser fluence from photoacoustic signals. Maslov et al. ${ }^{3}$ inserted a black object beneath the skin in animal models as a means of calibrating for relative laser fluence. Clearly, this method is not suitable for routine use on human subjects. Some groups have attempted to use diffuse optical tomography (DOT) as a second modality to estimate tissue properties and laser fluence variation. ${ }^{11}$ Unfortunately, this is not effective in the transport regime or the area close to the light source where photons do not undergo multiple scattering, and the spatial resolution of DOT may be suboptimal compared to photoacoustic methods. Other groups are attempting to use algorithms to iteratively or noniteratively use photoacoustic data to estimate local optical property variations quantitatively. Cox et al. ${ }^{12}$ showed that nonuniqueness issues plague such attempts. Zemp ${ }^{13}$ discussed a promising method using multiple optical sources, which may partially remedy this type of ill-posedness. Recently, Rajian and others ${ }^{14}$ injected contrast agents to compensate local laser fluence in blood oxygenation measurements.

In this study, we integrated an oblique incidence diffuse reflectance (OIR) technique with a photoacoustic probe. OIR is a quick, inexpensive, and noninvasive technique to measure bulk optical properties of a turbid medium. ${ }^{15}$ In OIR, the diffuse reflectance due to an obliquely angled laser beam incident on tissue is recorded (using a CCD camera) and analyzed to estimate the bulk optical properties. The basic principle of OIR is that the shift between the light entry point and the center of diffuse reflectance is a measure of the transport mean-free path (scaled by a multiplicative factor of the sine of the transmitted light entry angle). In addition, the natural

1083-3668/2010/15(4)/046016/5/\$25.00 @ 2010 SPIE 


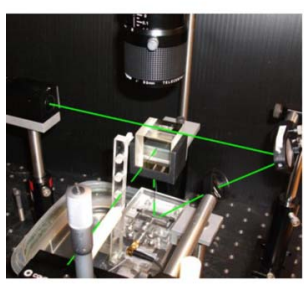

(a)

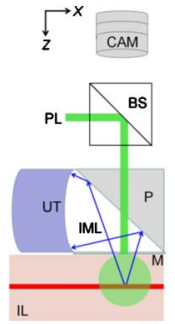

(b)

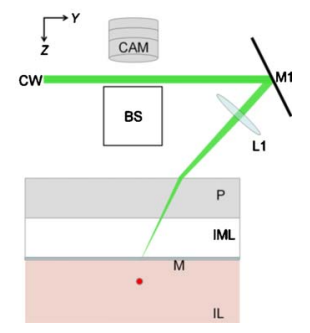

(c)
Fig. 1 Experimental setup: (a) Photograph of the setup. Overlaid solid green line shows the continuous wave $(\mathrm{CW})$ laser path, and dashed green line shows the pulsed laser $(\mathrm{PL})$ path; (b) photoacoustic mode; (c) OIR mode. CAM: camera; BS: beamsplitter; P: prism; IML: index matching liquid; M: membrane; IL: Intralipid; UT: ultrasound transducer; M1: mirror; L1: focusing lens. (Color online only.)

logarithm of far diffuse reflectance is proportional to the effective attenuation coefficient of the medium. A nonlinear curve fit of the diffuse reflectance to the diffusion theory model provides a measure of the effective attenuation coefficient. These parameters can be applied to obtain the reduced scattering coefficient and the absorption coefficient of the turbid medium. Once the optical parameters have been estimated, we can apply them in an appropriate light propagation model to calculate the local laser fluence at the absorber to normalize the photoacoustic measurements. This is the first report to our knowledge of combining OIR and photoacoustic methods to compensate the local laser fluence in the photoacoustic measurements.

\section{Method}

We modified a probe developed by our group ${ }^{16}$ previously. Briefly, the probe consisted of a prism, optical index-matching fluid, and an ultrasound transducer (Fig. 1). The indexmatching fluid enabled light to be directed downward undeflected, while ultrasound emerging from the sample due to photoacoustic excitation would travel upward through the index-matching fluid and be deflected by a prism to a sidelooking ultrasound transducer. We used a $10 \mathrm{~mm} \times 40 \mathrm{~mm}$ right-angle prism to allow space for an oblique light path. We used a $10 \mathrm{MHz}$ ultrasound transducer $(f=19.05 \mathrm{~mm}$, F\#3.1, spherical focus $\mathrm{G}$ series immersion type transducer, CD International Technology, Santa Clara, California). All parts were assembled in a custom-machined acrylic holder. Indexmatching liquid (Cat No. 19569, Cargille-Labs, Cedar Grove, New Jersey), which has a reflective index similar to fused silica prism $(n=1.46)$ was held underneath the prism by attaching a $\sim 25-\mu \mathrm{m}$-thick plastic wrap membrane to the holder. The index-matching fluid enabled light to be both delivered down to the sample and collected from the sample without appreciable refraction or reflection due to the prismtissue interface. A beamsplitter was mounted above the holder to divert a horizontal pulsed laser beam (Nd:YAG, Big Sky Technologies) downward [Fig. 1(b)]. The beam diameter at the membrane was $\sim 3.0 \mathrm{~mm}$. A long horizontal silicone dyefilled tube $(\mathrm{ID}=0.86 \mathrm{~mm}$; $\mathrm{OD}=1.5 \mathrm{~mm})$ mounted on an acrylic holder was immersed in an Intralipid (Intralipid 20\%, Fresenius Kabi, Uppsala, Sweden) bath underneath the laser interrogation point as a tissue and blood vessel mimicking phantom. The depth of the tube was adjusted by monitoring time of flight in ultrasound pulse-echo mode.

Based on the extinction coefficient at $532 \mathrm{~nm}$, six different concentrations of Crystal Violet dye (EMD Chemicals, Inc., Gibbstown, New Jersey) were chosen to have absorption coefficients of $25,50,75,100,125$, and $150 \mathrm{~cm}^{-1}$. The tube was filled with the absorbing dye during the experiment. It is worth mentioning that the tube was flushed with clean water before and after filling it with the dye. The tube was brought to the ultrasound focal region by translating it along the $y$ axis. To maintain the ultrasound focal axis and the optical axis of the laser in the same axis, the acrylic holder was translated along the $x$ axis until the maximum photoacoustic signal was observed. The maximum induced thermoelastic pressure rise, $p(z)$, at the tube immersed at depth $z$ is given by

$$
p(z)=\Gamma \Phi(z) \mu_{a \text { Dye }}+p_{\text {Tube }},
$$

where $\Gamma, \Phi(z), \mu_{a \text { Dye }}$, and $p_{\text {Tube }}$ are the Gruneisen parameter, the laser fluence at depth $z$, the absorption coefficients of Crystal Violet dye, and the thermoelastic pressure rise at the tube wall, respectively.

To obtain optical properties of Intralipid, we introduced OIR instrumentation. A CW-laser, a mirror, and a focusing lens $(f=35 \mathrm{~mm})$ were installed to provide a small spot at the membrane [Fig. 1(c)]. The CW laser beam interrogated the membrane at the same location as the pulsed laser used in the photoacoustic mode. Backscattered optical reflectance at the membrane was captured by a video camera (SI-SV5M10, Epix, Inc., Buffalo, Illinois) mounted above the beamsplitter. The data was processed to obtain reduced scattering coefficient and absorption coefficient of Intralipid. ${ }^{15}$ The laser fluence at depth $z$ was estimated by applying the preceding optical properties in a Monte Carlo simulation. When the amplitude of the photoacoustic signal is normalized by the local laser fluence, the modified pressure pressure rise $\left[p(z)_{\text {Modified }}\right]$ can be written as

$$
p(z)_{\text {Modified }}=k \mu_{a \text { Dye }}+k^{\prime},
$$

where $k$ and $k^{\prime}$ are constants. $p(z)_{\text {Modified }}$ is a linear function of the absorption coefficient of the dye. In other words, when the photoacoustic signal is normalized by the local laser fluence, it shows a linear relationship with an offset to the absorber concentration, irrespective of surrounding optical properties (unlike the unnormalized case).

We conducted two sets of experiments to demonstrate the relationship shown in Eq. (2). In the first experiment, we kept the absorber at a known depth and changed the turbid medium. We prepared five different Intralipid concentrations (IL1, IL2, IL3, IL4, and IL5) such that the concentrations of IL2, IL3, IL4, and IL5 were integer multiples of IL1. The tube filled with Crystal Violet dye of different absorption coefficients was immersed in an Intralipid bath. The corresponding photoacoustic signals were stored for each Intralipid concentration. In the second experiment, we selected one Intralipid concentration and changed the absorber depth. As the transducer response varies with the absorber depth, the photoacoustic signals must be normalized by the transducer response. We obtained the transducer response by measuring 


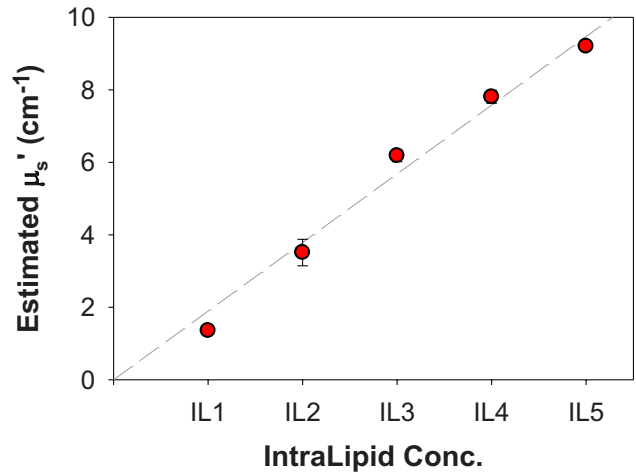

(a)

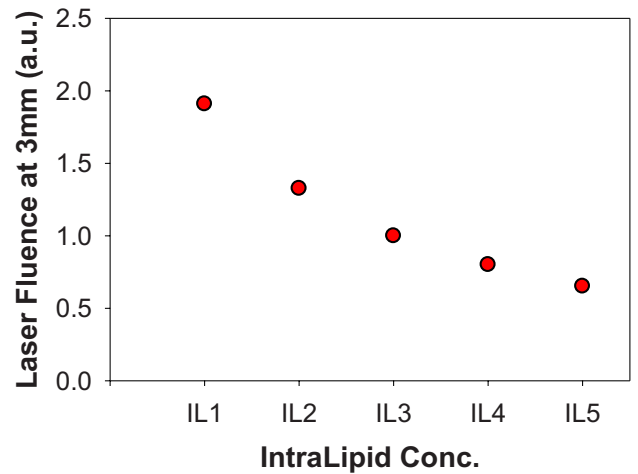

(b)

Fig. 2 (a) Reduced scattering coefficients obtained using the OIR technique, and (b) estimated laser fluences using the Monte Carlo simulation for various concentrations of Intralipid.

ultrasound signals of a human hair immersed in the water at different depths. The amplitude of the depth-dependent ultrasound signals are applied to normalize photoacoustic signals at different depths. Similar to the first experiment, the photoacoustic signals were recorded for different absorption coefficients of the dye. The OIR technique was used to obtain the optical properties for each Intralipid concentration. The results were applied in a Monte Carlo simulation to estimate the laser fluence at the absorber to normalize photoacoustic signals.

\section{Results}

The tube filled with the dye was kept at $3 \mathrm{~mm}$ below the membrane. Both photoacoustic and OIR measurements were obtained for each concentration of Intralipid. As we expected, the reduced optical scattering coefficients obtained from the OIR technique show a linear relationship with the concentration of Intralipid [Fig. 2(a)]. The laser fluences estimated by applying optical properties of Intralipid in Monte Carlo simulations are shown in Fig. 2(b).

The original photoacoustic signal amplitudes for different absorption coefficients of dye immersed in various concentra- tions of Intralipid are shown in Fig. 3(a). The result of normalizing photoacoustic signals by the local laser fluence [in Fig. 2(b)] is shown in Fig. 3(b). For one Intralipid concentration, depth of the tube $(d)$ was changed from 2.5 to $3.5 \mathrm{~mm}$ in steps of $0.5 \mathrm{~mm}$. Figure 4(a) shows the photoacoustic signals obtained for different dye concentrations. As the laser fluence at the absorber and transducer response change with the depth, Fig. 4(b) shows the data after normalizing the photoacoustic signals by the local laser fluence and the transducer response.

\section{Discussion}

We hypothesize that in many situations, bulk optical property characterization of tissues may be adequate to estimate local laser fluence to compensate photoacoustic measurements. While optical property inhomogeneities may confound the ultimate accuracy of this technique, we hypothesize that a firstorder fluence correction of photoacoustic signals is better than none at all. When the scattering coefficient of the turbid medium is increased, the local laser fluence at the absorber decreases [Fig. 2(b)]. As a result, the amplitude of the photoacoustic signal decreases with increasing Intralipid

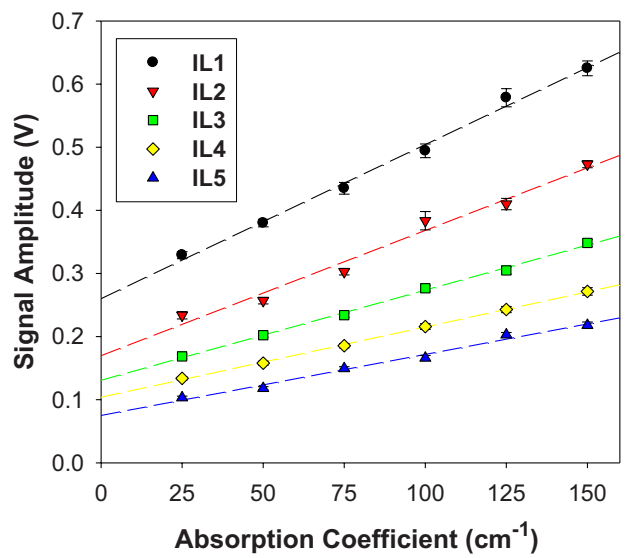

(a)

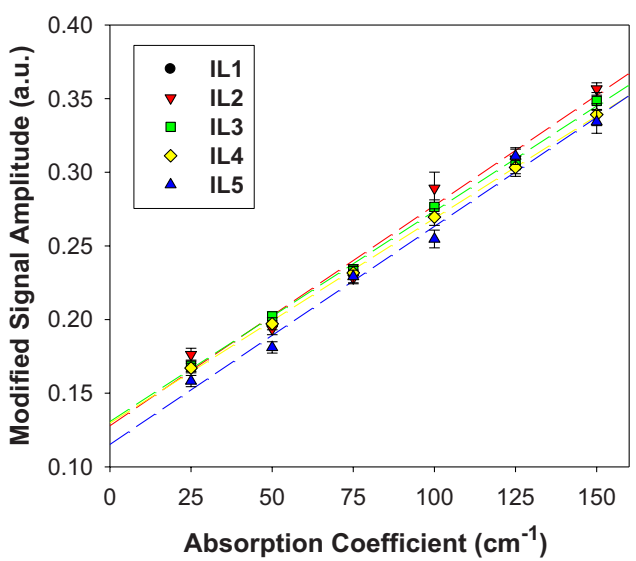

(b)

Fig. 3 Amplitude of photoacoustic signals obtained from a tube filled with various concentrations of dye. Each data set was obtained for different media (IL1, IL2, IL3, IL4, and IL5). The tube was held at constant depth (3 mm). (b) Modified photoacoustic signal versus absorption coefficient of the dye after normalizing by the local fluence. (Dashed lines depict the best-fit lines for each data set.) 


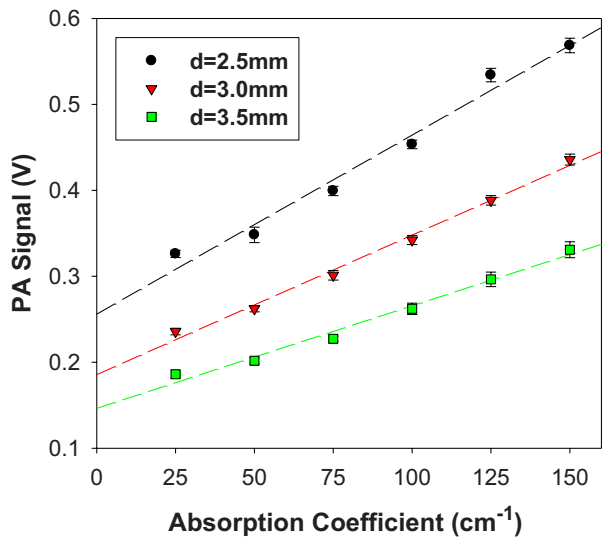

(a)

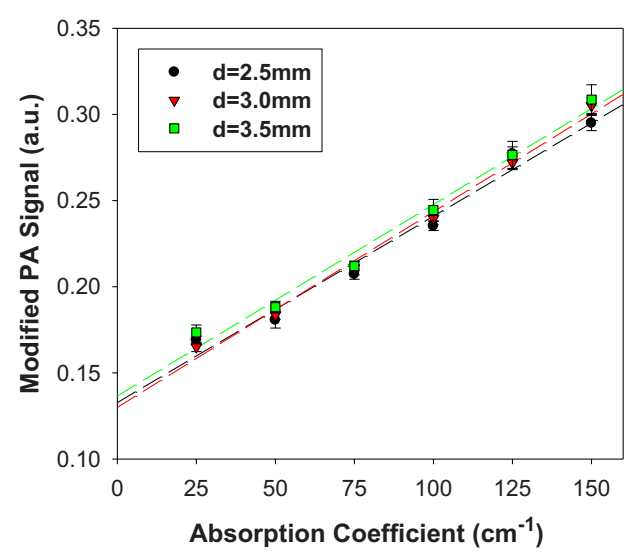

(b)

Fig. 4 (a) Photoacoustic signal obtained for a tube filled with dye having different absorption coefficients immersed at different depths (d). (b) Photoacoustic signals after normalizing by the local fluence and the transducer response. (Dashed lines depict the best-fit lines for each data set.)

concentration for each dye concentration. After normalizing by the local laser fluence, we can observe that data points with the same absorption coefficient fitted to a linear regression model provide an excellent $R^{2}(=0.99)$ value. This is important for quantitative comparison of an absorber concentration in various media. For example, when a photoacoustic molecular contrast agent is injected into an animal, this technique can be applied to compensate local laser fluence from photoacoustic signals obtained from various parts of the body. However, this technique may not be accurate for tissues with a greater variation of optical properties.

As the laser fluence at the absorber and transducer response change with the depth, the amplitude of the photoacoustic signal did not remain constant with unchanged dye concentration. For example, when the absorption coefficient of the dye is $150 \mathrm{~cm}^{-1}$, the amplitude of the photoacoustic signal was decreased by $\sim 42 \%$ for 1 -mm downward translation. After normalization by the local laser fluence, the difference between signals is less than 5\% [Fig. 4(b)]. All normalized data points fitted to a linear regression model show an outstanding fit $\left(R^{2}=0.99\right)$. As blood vessels are located at different depths, this technique can be applied to compensate the local laser fluence in photoacoustic signals. Then, modified photoacoustic signals can be potentially applied in quantitative photoacoustic imaging such as blood oxygenation and targeted molecular contrast agent imaging. In addition, this technique may be helpful in identifying amounts of laser energy that should be incident on the tissue for targeted photoacoustic drug delivery.

\section{Conclusion}

We have presented a combined photoacoustic oblique incidence reflectometry (OIR) system to compare absorber concentrations quantitatively. We obtained the OIR and photoacoustic measurements for different Intralipid concentrations and different absorber depths. The OIR measurements were applied to estimate the bulk optical parameters of the medium. Using these values, models of light transport were used to calculate the local laser fluence to normalize the photoacoustic measurements. The modified photoacoustic signals show linear relationships with the absorber dye concentrations. This technique may prove valuable for quantitative photoacoustic imaging in applications such as blood oxygenation estimation and targeted molecular imaging.

\section{Acknowledgments}

We acknowledge support from the Terry Fox Foundation through the Canadian Cancer Society (NCIC TFF 019237 and 019249), NSERC (G121211115), the Alberta Cancer Board (ACB 23728), Canada Foundation for Innovation Leaders Opportunity Fund, Alberta Advanced Education and Technology, and the University of Alberta for equipment and infrastructure. We also thank Dr. Robert Fedosejevs for sharing his $\mathrm{Nd}$ :YAG laser with our group.

\section{References}

1. J. Laufer, C. Elwell, D. Delpy, and P. C. Beard, "In vitro measurements of absolute blood oxygen saturation using pulsed near-infrared photoacoustic spectroscopy: accuracy and resolution," Phys. Med. Biol. 50(18), 4409-4428 (2005).

2. X. D. Wang, Y. J. Pang, G. Ku, X. Y. Xie, G. Stocia, and L. V. Wang, "Noninvasive laser-induced photoacoustic tomography for structural and functional in vivo imaging of the brain," Nat. Biotechnol. 21, 803-806 (2003).

3. K. Maslov, H. F. Zhang, and L. V. Wang, "Effects of wavelengthdependent fluence attenuation on the noninvasive photoacoustic imaging of hemoglobin oxygen saturation in subcutaneous vasculature in vivo," Inv. Prob. 23(6), S113-S122 (2007).

4. J. M. Murkin, S. J. Adams, R. J. Novick, M. Quantz, D. Bainbridge, I. Iglesias, A. Cleland, B. Schaefer, B. Irwin, and S. Fox, "Monitoring brain oxygen saturation during coronary bypass surgery: a randomized, prospective study," Anesth. Analg. 104(1), 51-58 (2007).

5. C. Menon and D. L. Fraker, "Tumor oxygenation status as a prognostic marker," Cancer Lett. 221(2), 225-235 (2005).

6. A. A. Tandara and T. A. Mustoe, "Oxygen in wound healing-more than a nutrient," World J. Surg. 28(3), 294-300 (2004).

7. A. De la Zerda, Z. Liu, C. Zavaleta, S. Bodapati, R. Teed, S. Vaithilingam, T. Ma, O. Oralkan, X. Chen, B. T. Khuri-Yakub, H. Dai, and S. S. Gambhir, "Enhanced sensitivity carbon nanotubes as targeted photoacoustic molecular imaging agents," Proc. SPIE 7177, 1-8 (2009).

8. L. Xiang, Y. Yuan, D. Xing, Z. Ou, S. Yang, and F. Zhou, "Photoacoustic molecular imaging with antibody-functionalized singlewalled carbon nanotubes for early diagnosis of tumor," J. Biomed. Opt. 14, 021008 (2009).

9. K. Kim, S. Huang, S. Ashkenazi, M. O’Donnell, A. Agarwal, N. A. Kotov, N. F. Denny, and M. J. Kaplan, "Photoacoustic imaging of early inflammatory response using gold nanorods," Appl. Phys. Lett. 
90, 223901 (2007)

10. S. Mallidi and S. Emelianov, "Photoacoustic technique to measure beam profile of pulsed laser systems," Rev. Sci. Instrum. 80(5), 054901 (2009)

11. L. Yin, Q. Wang, Q. Zhang, and H. Jiang, "Tomographic imaging of absolute optical absorption coefficient in turbid media using combined photoacoustic and diffusing light measurements," Opt. Lett. 32, 2556-2558 (2007).

12. B. T. Cox, S. R. Arridge, and P. C. Beard, "Estimating chromophore distributions from multiwavelength photoacoustic images," J. Opt. Soc. Am. A 26, 443-455 (2009).
13. R. J. Zemp, "Quantitative photoacoustic tomography with multiple optical sources," Appl. Opt. 49, 3566-3572 (2010).

14. J. R. Rajian, P. L. Carson, and X. Wang, "Quantitative photoacoustic measurement of tissue optical absorption spectrum aided by an optical contrast agent," Opt. Express 17(6), 4879-4889 (2009).

15. S. Lin, L. H. Wang, S. L. Jacques, and F. K. Tittel, "Measurement of tissue optical properties by the use of oblique-incidence optical fiber reflectometry," Appl. Opt. 36, 136-143 (1997).

16. R. J. Zemp, J. Ranasinghesagara, Y. Jiang, X. Chen, and K. Mathewson, "A photoacoustic method for optical scattering measurements in turbid media," Proc. SPIE 7177, 71770Q (2009). 